\title{
First principles study of the multiferroics $\mathrm{BiFeO}_{3}, \mathrm{Bi}_{2} \mathrm{FeCrO}_{6}$, and $\mathrm{BiCrO}_{3}$ : Structure, polarization, and magnetic ordering temperature
}

\author{
Pio Baettig,* Claude Ederer, and Nicola A. Spaldin \\ Materials Department and Materials Research Laboratory, University of California, Santa Barbara, California 93106, USA
}

(Received 23 August 2005; revised manuscript received 19 October 2005; published 6 December 2005)

\begin{abstract}
We present results of an ab initio density-functional theory study of three bismuth-based multiferroics, $\mathrm{BiFeO}_{3}, \mathrm{Bi}_{2} \mathrm{FeCrO}_{6}$, and $\mathrm{BiCrO}_{3}$. We disuss differences in the crystal and electronic structure of the three systems and show that the application of the LDA $+U$ method is essential to obtain realistic structural parameters for $\mathrm{Bi}_{2} \mathrm{FeCrO}_{6}$. We calculate the magnetic nearest-neighbor coupling constants for all three systems and show how Anderson's theory of superexchange can be applied to explain the signs and relative magnitudes of these coupling constants. From the coupling constants we then obtain a mean-field approximation for the magnetic ordering temperatures. Guided by our comparison of these three systems, we discuss the possibilities for designing a multiferroic material with large magnetization above room temperature.
\end{abstract}

DOI: 10.1103/PhysRevB.72.214105

PACS number(s): 77.80.-e, 75.30.Et, 71.15.Mb

\section{INTRODUCTION}

There has been growing recent interest in the magnetic, ferroelectric, and piezoelectric properties of bismuth-based perovskite-structure oxides. ${ }^{1-10}$ In such materials, the formally $\mathrm{Bi}^{3+}$ ion occupies the perovskite $A$ site, and its stereochemically active $6 s^{2}$ lone pair induces a symmetrylowering structural distortion that can lead to ferroelectricity.

2 Part of this interest lies in increasing concern about the environmental toxicity of lead-based piezoelectrics and the resulting search for alternative materials. ${ }^{10,11}$ In addition, the stereochemical activity of $\mathrm{Bi}$ is being exploited to induce structural distortions in magnetic oxides, with the goal of forming ferromagnetic ferroelectrics (so-called magnetoelectric multiferroics) ${ }^{1-9}$ Although a large number of potential applications can be envisaged for a material that is simultaneously ferromagnetic and ferroelectric, ${ }^{12}$ there are currently no known single-phase materials that show large, robust magnetization and polarization at room temperature.

In this work we analyze the magnetic properties of three related Bi-based multiferroics in order to understand the chemical and structural factors that affect the magnetic ordering temperature. Our goal is to develop guidelines for designing new perovskite-structure ferroelectrics with a large macroscopic magnetization above room temperature. The focus of our investigation is the (111) layered double perovskite $\mathrm{Bi}_{2} \mathrm{FeCrO}_{6}$, and its parent compounds $\mathrm{BiFeO}_{3}$ and $\mathrm{BiCrO}_{3} . \mathrm{BiFeO}_{3}$ is the most well studied of the three compounds and has long been known to be ferroelectric, with a Curie temperature $T_{C} \sim 1100 \mathrm{~K}$, and antiferromagnetic, with a Néel temperature $T_{N} \sim 640 \mathrm{~K} \cdot{ }^{13,14}$ It crystallizes in a rhombohedrally distorted perovskite structure with space group $R 3 c{ }^{15}$ Recently, interest in this material has increased considerably, due to the report of a large electric polarization and a nonzero magnetization in epitaxial films of $\mathrm{BiFeO}_{3}$, both above room temperature. ${ }^{4}$ The large electric polarization, as well as the small magnetization found in 200-400 nm thick $\mathrm{BiFeO}_{3}$ films, have been confirmed by other experiments and by first-principles calculations ${ }^{7-9}$ (see also Sec. III C), whereas the origin of the large magnetiza- tion observed for film thickness $<100 \mathrm{~nm}$ is still under debate.

$\mathrm{BiCrO}_{3}$ was recently synthesized and reported to be a highly distorted perovskite with $C 2$ symmetry, ${ }^{5}$ a polar space group that permits the occurrence of ferroelectricity. Antiferromagnetic ordering was reported with a residual magnetization of $0.02 \mu_{B}$ per $\mathrm{Cr}$, suggestive of weak ferromagnetism.

$\mathrm{Bi}_{2} \mathrm{FeCrO}_{6}$ has not yet been realized experimentally, but has been predicted theoretically to be ferrimagnetic (with a magnetic moment of $2 \mu_{B}$ per formula unit) and ferroelectric (with a polarization of $\sim 80 \mu \mathrm{C} / \mathrm{cm}^{2}$ ). ${ }^{6}$ The predicted ground state structure is very similar to the $R 3 c$ structure of $\mathrm{BiFeO}_{3}$, except that in every second (111) layer the $\mathrm{Fe}^{3+}$ cations are replaced by $\mathrm{Cr}^{3+}$, which reduces the symmetry to the space group $R 3$. Such a $B$-site-ordered structure could be realized by (111) layer-by-layer growth on an appropriate substrate. ${ }^{16}$

The remainder of this article is structured as follows: First we present the computational details of our calculations. Then, in Sec. III A-III C, we compare the calculated ground state structures of the three systems, with a particular focus on the influence of the exchange-correlation potential used in the density-functional formalism on the structural parameters; this is particularly important for reproducing the correct physics in these strongly correlated magnetic insulators. We also compare the calculated electric polarization of the three materials. In Sec. III D, we calculate nearest- and nextnearest-neighbor magnetic couplings and determine the magnetic ordering temperatures for the three materials using the mean-field approximation. Finally, in Sec. IV, we discuss the variation of the nearest-neighbor couplings in these systems and the implications of our results for the design of new multiferroics with higher magnetic Curie temperatures.

\section{COMPUTATIONAL DETAILS}

All calculations described in this work were performed using the projector-augmented wave (PAW) formalism ${ }^{17}$ of density-functional theory, ${ }^{18,19}$ implemented in the Vienna Ab Initio Simulation Package (VASP). ${ }^{20,21}$ For $\mathrm{BiFeO}_{3}$ the $\mathrm{Bi} 5 d$ and the Fe $3 p$ semicore states were treated as valence elec- 
trons. However, since test calculations showed that the inclusion of these states has no significant effect on the properties relevant for this work, we did not include semicore states in the valence for $\mathrm{Bi}_{2} \mathrm{FeCrO}_{3}$ and $\mathrm{BiCrO}_{3}$. The local spindensity approximation (LDA) as well as the LDA+ $U$ method in the so-called fully localized limit $^{22}$ was used to treat exchange and correlation.

Application of the LDA in the case of transition metal oxides usually leads to a severe underestimation of the band gap and in some cases even predicts metallic behavior for systems that are known to be insulators. ${ }^{23}$ This deficiency is due to the mean field character of the LDA, which results in an inability to describe orbital polarization effects, expecially the splitting between filled and unfilled orbitals in MottHubbard-type insulators. The LDA $+U$ method improves on this deficiency by adding an additional term to the total energy of the system, which explicitely treats correlation effects between certain localized orbitals. The parameter $U$ gives the strength of the Coulomb repulsion between these orbitals, and an additional parameter, $J$, describes the on-site exchange interaction between these orbitals. The LDA $+U$ method has been successfully applied to describe the electronic structure of systems containing localized $d$ and $f$ electrons where LDA leads to qualitatively wrong results, ${ }^{22}$ and recently it has also been applied to obtain structural parameters that are in better agreement with experimental data than the corresponding LDA results. $7,24,25$

In this work, except where otherwise noted, the structural parameters were fixed to those obtained by full structural optimization using $U=3 \mathrm{eV}$ and $J=0.8 \mathrm{eV}$ (for $\mathrm{Bi}_{2} \mathrm{FeCrO}_{6}$ and $\mathrm{BiCrO}_{3}$ ) or $J=1 \mathrm{eV}$ (for $\mathrm{BiFeO}_{3}$ ) for the treatment of the transition metal $d$ orbitals. Then, the electronic structure and magnetic coupling constants were calculated as a function of $U$ (and fixed $J$ ) in the range $U=3 \mathrm{eV}-6 \mathrm{eV}$. For simplicity, the same $U$ and $J$ were used on both the $\mathrm{Fe}$ and $\mathrm{Cr}$ sites in $\mathrm{Bi}_{2} \mathrm{FeCrO}_{6}$. All our results are well converged with respect to $k$-point mesh and the energy cutoff for the plane wave expansion. For details regarding the structural optimization of $\mathrm{BiFeO}_{3}$, see Ref. 7, and Ref. 6 for $\mathrm{Bi}_{2} \mathrm{FeCrO}_{6}$. The electric polarization was calculated using the Berry phase method. ${ }^{26,27}$

\section{RESULTS AND DISCUSSION}

\section{A. Ground-state structures and magnetic orderings}

We begin by comparing the calculated structures of the three compounds. The ground-state structure of $\mathrm{Bi}_{2} \mathrm{FeCrO}_{6}$ was calculated in Ref. 6 by optimizing the geometries of a range of starting configurations, obtained by freezing in high-symmetry unstable phonon modes of $\mathrm{BiCrO}_{3}{ }^{3}$ and $\mathrm{BiAlO}_{3} .{ }^{10}$ The energies of structures constrained to the resulting symmetries are listed in Table I for two magnetic configurations: ferromagnetic, and the ferrimagnetic equivalent of so-called $G$-type antiferromagnetic ordering, in which all spins within the same (111) plane are ferromagnetically aligned, with antiparallel alignment of spins in adjacent (111) planes. We see that the lowest energy structure of all the combinations tried has $R 3$ symmetry (space group 146), with alternating rotations of the oxygen octahedra around the
TABLE I. Total energies per formula unit of $\mathrm{Bi}_{2} \mathrm{FeCrO}_{6}$, for different structural symmetries relative to the ground-state structure for ferromagnetic (FM) and ferrimagnetic (FiM) ordering, respectively.

\begin{tabular}{llc}
\hline \hline Space group & Magnetic ordering & $\begin{array}{c}E \\
{[\mathrm{eV} / \mathrm{f} . \mathrm{u} .]}\end{array}$ \\
\hline$P m \overline{3} m$ & FM & 1.722 \\
& FiM & 1.670 \\
Cmca & FM & 1.215 \\
& FiM & 1.096 \\
& FM & FiM \\
\multirow{2}{*}{ R3m } & FM & 0.837 \\
& FiM & 0.683 \\
& FM & 0.612 \\
& FiM & 0.473 \\
\hline \hline
\end{tabular}

[111] direction, combined with relative displacements of all ions along [111]. This structure is closely related to the $R 3 \mathrm{c}$ structure found experimentally for $\mathrm{BiFeO}_{3},{ }^{15}$ but with an additional symmetry lowering due to the different $B$ cations. From Table I one can also see that the ferrimagnetic configuration has a lower energy than the ferromagnetic case for all structural symmetries. The net magnetic moment of the ferrimagnetic case is $2 \mu_{B}$ per $\mathrm{Fe}-\mathrm{Cr}$ pair, which corresponds to a magnetization of $160.53 \mathrm{emu} / \mathrm{cm}^{3}$ in the case of the ground-state $R 3$ structure. It is also clear from Table I that typical energy differences between different magnetic configurations are significantly smaller than the energy differences due to different structural symmetries. Moreover, the structural parameters (not shown) obtained for FM and FiM ordering within a given structural symmetry differ only slightly.

To exclude the existence of a more complicated magnetic configuration with lower energy, we doubled the size of the unit cell along one of the rhombohedral lattice vectors and compared the total energies for all possible collinear spin configurations within the resulting supercell. The doubled unit cell allows for spin configurations with antiparallel alignment of magnetic cations of the same type, which would lead to a cancellation of the macroscopic magnetization. For these calculations no additional structural relaxations were performed; the structure was fixed to that obtained by relaxation within the ferrimagnetic $R 3$ symmetry. Based on the very similar structures we obtained above for FM and FiM orderings, we do not expect that further structural relaxation would alter the relative energies of the different spin configurations. It was found that the " $G$-type-like" ferrimagnetic configuration described above is indeed the ground state, and no cancellation of the macroscopic magnetization occurs. We did not investigate the effect of spin-orbit coupling and the possibility of noncollinear spin structures or long-wavelength magnetic ordering.

For $\mathrm{BiFeO}_{3}$ both the structure and the magnetic configuration are well established. ${ }^{15,28}$ Therefore, we did not perform a similar thorough ground state search for this system 
TABLE II. Calculated lattice constant $a$, rhombohedral angle $\alpha$, volume $V$, and Wyckoff parameters for $\mathrm{BiCrO}_{3}, \mathrm{Bi}_{2} \mathrm{FeCrO}_{6}$, and $\mathrm{BiFeO}_{3}$. The internal structural parameters refer to the Wyckoff positions $2 a(x, x, x)$ for the cations and $6 b(x, y, z)$ for the anions (in case of the $R 3$ structure the corresponding Wyckoff labels are $1 a$ and $3 b)$.

\begin{tabular}{lcccc}
\hline \hline & & $\mathrm{BiCrO}_{3}$ & $\mathrm{Bi}_{2} \mathrm{FeCrO}_{6}$ & $\mathrm{BiFeO}_{3}$ \\
\hline$a[\AA]$ & & 5.43 & 5.47 & 5.50 \\
$\alpha\left[^{\circ}\right]$ & & 60.37 & 60.09 & 59.99 \\
$V\left[^{\circ} \AA^{3}\right]$ & & 114.47 & 116.86 & 117.86 \\
$\mathrm{Bi}$ & $x$ & 0.000 & $0.000 / 0.503$ & 0.000 \\
$\mathrm{Cr} / \mathrm{Fe}$ & $x$ & 0.228 & $0.226(\mathrm{Cr}) / 0.732(\mathrm{Fe})$ & 0.228 \\
$\mathrm{O}$ & $x$ & 0.546 & $0.545 / 0.047$ & 0.542 \\
& $y$ & 0.952 & $0.950 / 0.905$ & 0.942 \\
& $z$ & 0.407 & $0.398 / 0.448$ & 0.368 \\
\hline \hline
\end{tabular}

and instead only relaxed the structure within the experimentally found $R 3 c$ symmetry in combination with $G$-type antiferromagnetic ordering. The details of the structural relaxation of $\mathrm{BiFeO}_{3}$ have already been presented in Ref. 7, and all calculated structural parameters agree well with experimental data. We note that the true magnetic structure of $\mathrm{BiFeO}_{3}$ shows some slight deviations from the ideal $G$-type ordering, i.e., a long-wavelength spiral structure in the bulk ${ }^{29}$ and a slight canting of the magnetic moments in thin films, ${ }^{8,9}$ both caused by spin-orbit coupling. However, these effects are rather small and not relevant for the present study.

In the case of $\mathrm{BiCrO}_{3}$ we also constrained the system to have rhombohedral $R 3 c$ symmetry and $G$-type antiferromagnetic ordering in our calculations, although recently this system was reported to have monoclinic symmetry. ${ }^{5}$ This was done in order to be able to systematically compare the properties of the series of compounds $\mathrm{BiFeO}_{3}, \mathrm{Bi}_{2} \mathrm{FeCrO}_{6}$, and $\mathrm{BiCrO}_{3}$.

The calculated lattice constants, rhombohedral angles, unit cell volumes, and internal structural parameters for all three systems are summarized in Table II. It can be seen that the volume and lattice constants increase over the series $\mathrm{BiCrO}_{3}, \mathrm{Bi}_{2} \mathrm{FeCrO}_{6}, \mathrm{BiFeO}_{3}$, due to the larger radius of the high-spin $\mathrm{Fe}^{3+}$ ion compared to $\mathrm{Cr}^{3+}$ (see also Sec. III B). Likewise the rhombohedral angle is reduced. The internal structural parameters, i.e., the positions of the atoms within the unit cell are very similar in all three systems. This partly reflects the fact that $\mathrm{Bi}$ is the "active" ion causing the ferroelectric distortion.

\section{B. Effect of $U$ on the structure of $\mathrm{Bi}_{2} \mathrm{FeCrO}_{6}$}

There is an interesting subtlety involved in the structural relaxation of the $\mathrm{Bi}_{2} \mathrm{FeCrO}_{6}$ system, regarding the influence of the parameter $U$ on the ground-state structural parameters. Whereas LDA $(U=0)$ leads to a metallic low-spin state of the $\mathrm{Fe}^{3+}$ cation (albeit not changing the fact that the ground state is ferrimagnetic with $R 3$ symmetry), the use of a moderate $U$ leads to an insulating solution containing high-spin $\mathrm{Fe}^{3+}$. Because of the substantial differences in the ionic radii

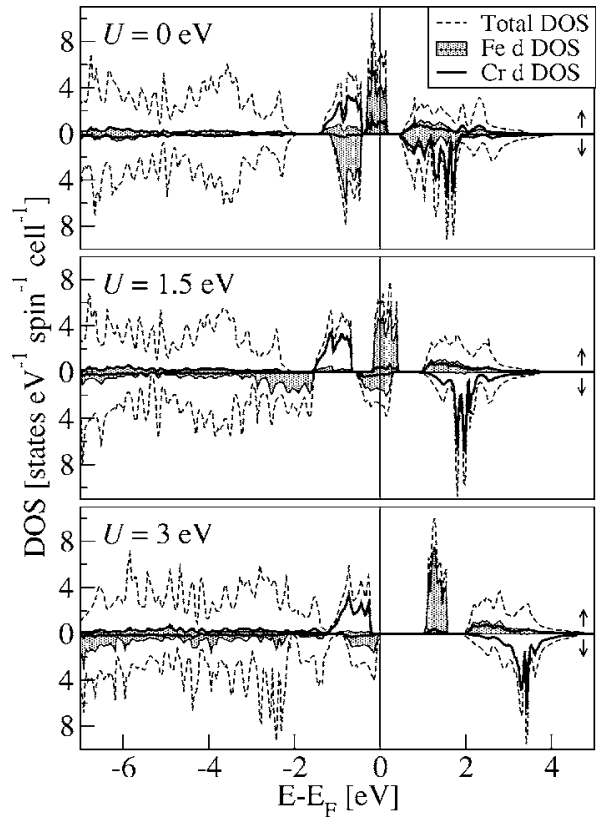

FIG. 1. Spin-resolved total and transition metal $d$ densities of states (DOS) for $U=0.0 \mathrm{eV}$ (LDA), $1.5 \mathrm{eV}$ and $3.0 \mathrm{eV}$. The total DOS is indicated by the dashed lines, the Fe $d$ DOS by shading and the $\mathrm{Cr} d$ DOS by thick lines.

of low-spin versus high-spin $\mathrm{Fe}^{3+}$ (the Shannon ionic radii are 0.55 and $0.65 \AA$, respectively ${ }^{30}$ ), this has a significant effect on the lattice parameters. Using LDA for the structural relaxation we obtain a lattice constant of $a=5.30 \AA$, a rhombohedral angle $\alpha=61.23^{\circ}$, and an equilibrium volume that is $\sim 7 \%$ smaller than that obtained with $\mathrm{LDA}+U$ and $U$ $=3 \mathrm{eV}$.

The evolution of the electronic structure with increasing $U$ is illustrated in Fig. 1, which shows our calculated spinresolved densities of states for $U=0$ (the LDA result), $1.5 \mathrm{eV}$, and $3 \mathrm{eV}$ ( $R 3$ symmetry, FiM). To isolate differences in electronic properties from structural effects, the same (LDA) structure is used in all cases. The orbital-resolved densities of states are obtained by projection onto a local angular-momentum basis and integrating up to a radius of $1.4 \AA$. In all cases, the spin of the $\mathrm{Cr}$ ion defines the "upspin" direction, and its occupied $\left(t_{2 g}^{\uparrow}\right)^{3}$ manifold can be seen on the positive $y$ axis just below the Fermi energy. In the LDA solution, the Fe $t_{2 g}^{\downarrow}$ manifold is completely filled, and the $t_{2 g}^{\uparrow}$ states are partially filled, leading to a finite density of states at the Fermi energy. The calculated local magnetic moments (also calculated by integrating up to a Wigner-Seitz radius of $1.4 \AA$ ) on the $\mathrm{Cr}$ and $\mathrm{Fe}$ ions are $+2.5 \mu_{B}$ and $-0.6 \mu_{B}$, respectively. These values are slightly reduced from the "ideal" values of +3 and -1 expected for $\mathrm{Cr}^{3+}$ and lowspin $\mathrm{Fe}^{3+}$ because of hybridization with the oxygen band and the certain degree of arbitrariness in the definition of the Wigner-Seitz spheres. Whereas the positions of the $\mathrm{Cr}$ states do not change significantly as a function of $U$, the Fe states are strongly affected by application of a small $U$ of $1.5 \mathrm{eV}$; the $t_{2 g}^{\downarrow}$ states shift down in energy and hybridize with the oxygen $p$ band ( $\sim 2 \mathrm{eV}$ below the Fermi energy), and the $e_{g}^{\downarrow}$ states shift down into the same energy region as the $t_{2 g}^{\uparrow}$ 
states, and become partially occupied. On increasing $U$ to $3 \mathrm{eV}$, the Fe $e_{g}^{\downarrow}$ states become fully occupied, the $t_{2 g}^{\uparrow}$ states are completely empty, and an insulating solution is obtained. The calculated local magnetic moments are now $+2.5 \mu_{B}$ for the $\mathrm{Cr}^{3+}$ ion (unchanged from the LDA value) and $-4.0 \mu_{B}$ for the $\mathrm{Fe}^{3+}$, again slightly reduced from the ideal high-spin value of $-5.0 \mu_{B}$. Further increase of $U$ does not change the electronic structure qualitatively.

The above result shows that for $\mathrm{Bi}_{2} \mathrm{FeCrO}_{6}$ the application of the $\mathrm{LDA}+U$ method induces a major change in the electronic structure which, in turn, has a pronounced effect on the structural parameters. Traditionally, the LDA $+U$ method has often been used in combination with the atomic sphere approximation (ASA), ${ }^{31}$ which does not allow structural relaxation. Only recently has the $\mathrm{LDA}+U$ method also been applied to obtain structural parameters, in general with considerable success (see, e.g., Refs. 7, 24, and 25). It is reasonable to assume that the electronic structure of $\mathrm{Bi}_{2} \mathrm{FeCrO}_{6}$ resembles much more the situation found for $U$ $=3 \mathrm{eV}$, with a pronounced gap and high-spin $\mathrm{Fe}^{3+}$, than the pure LDA solution (which is metallic and contains low-spin $\mathrm{Fe}^{3+}$ ). Therefore, we predict that the structural parameters obtained for $U=3 \mathrm{eV}$ will be much closer to experimentally obtained structural parameters than the LDA results. Our results show that, in situations where the application of the $\mathrm{LDA}+U$ method can change the spin state of an ion, this latter approach is essential for a meaningful result to be obtained.

Interestingly, if we relax the structural parameters using LDA, but fix the total magnetic moment to be $8 \mu_{B}$ (corresponding to high-spin states for both $\mathrm{Cr}$ and $\mathrm{Fe}$ and a ferromagnetic configuration of all spins), the resulting structure is very similar to that obtained by using $\mathrm{LDA}+U$ with $U$ $=3 \mathrm{eV}$. This shows that the main effect of the on-site Coulomb repulsion in $\mathrm{Bi}_{2} \mathrm{FeCrO}_{6}$ on the structure is the stabilization of the high-spin state of the $\mathrm{Fe}^{3+}$ cation. The larger volume of this high-spin state then determines the structure. On the other hand, as long as the $\mathrm{Fe}^{3+}$ cation is in its highspin state, the precise value of $U$ does not have a pronounced effect on the structural parameters. As a consequence, the calculated LDA structure for $\mathrm{BiFeO}_{3}$ is very similar to the corresponding LDA $+U$ structure, because in both cases the transition metal ion adopts the same spin configuration. ${ }^{7}$

\section{Calculated polarization}

Table III shows the spontaneous electric polarization $P_{s}$ for the three systems, calculated with the Berry-phase method (see Sec. II), as well as an estimate for $P_{s}$, calculated by multiplying the ferroelectric displacements, $u_{i}$, by the formal ionic charges, $Z_{i}$, and summing up over all ions $i$. It can be seen that, for both methods, the polarization increases from $\mathrm{BiCrO}_{3}$ to $\mathrm{Bi}_{2} \mathrm{FeCrO}_{6}$ to $\mathrm{BiFeO}_{3}$, reflecting a corresponding increase in ferroelectric displacements of the ions. The polarization calculated from the Berry phase is consistently larger than the simple estimate by a factor of $\sim 1.5$. This represents the well-known fact that the effective charges in perovskite ferroelectrics are usually significantly larger than the formal charges. [For example, the effective charges
TABLE III. Spontaneous polarization $P_{s}$ calculated with the Berry phase method for all three systems, together with a simple estimate, obtained by summing up formal charges $Z_{i}$ times displacements $u_{i}$ for all ions $i$ in the unit cell (values in $\mu \mathrm{C} / \mathrm{cm}^{2}$ ). The ratio between these two quantities is also given.

\begin{tabular}{lccc}
\hline \hline & $P_{s}$ & $\sum_{i} Z_{i} u_{i}$ & $P_{s} / \Sigma_{i} Z_{i} u_{i}$ \\
\hline $\mathrm{BiCrO}_{3}$ & 67 & 46 & 1.46 \\
$\mathrm{Bi}_{2} \mathrm{FeCrO}_{6}$ & 80 & 55 & 1.45 \\
$\mathrm{BiFeO}_{3}$ & 95 & 64 & 1.48 \\
\hline \hline
\end{tabular}

in ground-state $R 3 c \mathrm{BiFeO}_{3}$ are 4.4, 3.5, and -2.5 for $\mathrm{Bi}, \mathrm{Fe}$, and $\mathrm{O}$, respectively (see Ref. 7), whereas the corresponding formal charges are 3.0, 3.0, and -2.0$]$. The nearly constant ratio between the simple estimate and the Berry phase $P_{s}$, indicates that the effective charges are comparable in all cases.

The polarizations of all three systems are relatively large and comparable to that of $\mathrm{PbTiO}_{3}\left(75 \mu \mathrm{C} / \mathrm{cm}^{2}\right){ }^{32}$ This suggests that $\mathrm{Bi}$-based perovskite ferroelectrics could be promising candidates for alternative "Pb-free" ferroelectric and piezoelectric devices. ${ }^{10}$

\section{Magnetic ordering temperatures and exchange couplings}

To estimate the magnetic ordering temperature for the newly predicted multiferroic $\mathrm{Bi}_{2} \mathrm{FeCrO}_{6}$, we determine the Heisenberg exchange constants corresponding to nearestand next-nearest-neighbor magnetic couplings by comparing the total energies for different magnetic configurations. From these coupling constants we then calculate the magnetic ordering temperatures within the mean-field approximation (see, e.g., Ref. 33). For comparison, we perform analogous calculations for $\mathrm{BiFeO}_{3}$ and $\mathrm{BiCrO}_{3}$. In the mean-field approximation of the Heisenberg model the interactions between the different spins are described by an effective field that is proportional to the expectation value of the magnetic order parameter. The mean-field approximation therefore neglects the effect of fluctuations of the spins from their average values. Since, in general, such fluctuations tend to decrease the magnetic ordering temperature, the mean-field approximation gives an upper limit of the actual ordering temperature.

To obtain the Heisenberg coupling constants for nearestand next-nearest-neighbor coupling, we double the size of the unit cell (so that it contains two formula units of $\mathrm{Bi}_{2} \mathrm{FeCrO}_{6}$ ) and calculate the total energies for several possible inequivalent collinear magnetic configurations with different orientations of the magnetic moments on different sites. We then map the calculated energies onto a simple Heisenberg model, $E=-\frac{1}{2} \sum_{i j} J_{i j} S_{i} S_{j}$. Here $S_{i}= \pm \frac{3}{2}$ on the $\mathrm{Cr}$ sites and $\pm \frac{5}{2}$ on the Fe sites, and the sum is over all nearestand next-nearest-neighbor pairs. All calculated total energies can be reproduced accurately using only nearest- and nextnearest-neighbor couplings.

In Refs. 34 and 35, the coupling constants for $\mathrm{Sr}_{2} \mathrm{FeMO}_{6}$ $(M=W, \mathrm{Mo}, \mathrm{Re})$ and for $\mathrm{BaFe}_{12} \mathrm{O}_{19}$ were found to be rather sensitive to the value of $U$. This reflects the dominant role of 


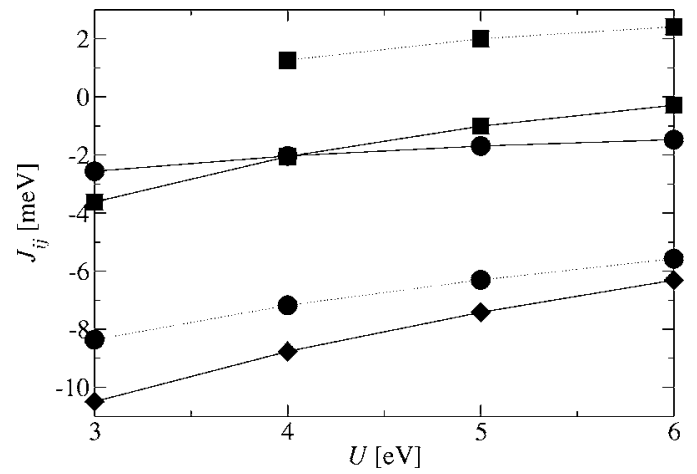

FIG. 2. Nearest-neighbor coupling constants, $J_{i j}$, for $\mathrm{BiFeO}_{3}$, $\mathrm{Bi}_{2} \mathrm{FeCrO}_{6}$, and $\mathrm{BiCrO}_{3}$ in their ground-state structures as well as the corresponding coupling constants for $\mathrm{Bi}_{2} \mathrm{FeCrO}_{6}$ and $\mathrm{BiCrO}_{3}$ in the ideal cubic perovskite structure, as a function of $U$. Dashed lines are for the cubic structures; full lines are for the relaxed structures. Circles are for $\mathrm{BiCrO}_{3}$, squares for $\mathrm{Bi}_{2} \mathrm{FeCrO}_{6}$, and diamonds for $\mathrm{BiFeO}_{3}$.

the superexchange interaction in magnetic oxides, in which the antiferromagnetic coupling is proportional to $b^{2} / U$, where $b$ is the transfer integral describing hybridization effects. ${ }^{36}$ We therefore calculate all coupling constants as a function of $U$ in the range $3-6 \mathrm{eV}$ using the structural parameters shown in Table II. $J$ was set to $1 \mathrm{eV}$ for all systems. To separate out effects of changing structure from differences in chemistry, we also calculate the exchange couplings for ideal cubic perovskite $\mathrm{BiCrO}_{3}, \mathrm{BiFeO}_{3}$, and $\mathrm{Bi}_{2} \mathrm{FeCrO}_{6}$, at the same average volume of $116.2 \AA^{3}$. Figure 2 shows the nearest-neighbor coupling constants for all three relaxed systems and for perfect cubic $\mathrm{BiCrO}_{3}$ and $\mathrm{Bi}_{2} \mathrm{FeCrO}_{6}$ as a function of $U$. (For cubic $\mathrm{BiFeO}_{3}$ some magnetic configurations are metallic up to $U=6 \mathrm{eV}$. Since in the metallic state different magnetic coupling mechanisms are present, we do not include the values for cubic $\mathrm{BiFeO}_{3}$ in our systematic comparison.) For both cubic and relaxed structures we obtain a reduction in the strength of the antiferromagnetic superexchange as $U$ is increased (Fig. 2); indeed for cubic $\mathrm{Bi}_{2} \mathrm{FeCrO}_{6}$ the nearest-neighbor coupling even becomes ferromagnetic.

From the coupling constants $J_{i j}$ we calculate the magnetic ordering temperature using the mean-field approximation (see, e.g., Ref. 33). Figure 3 shows the resulting magnetic ordering temperatures for all three systems. Comparing the values of $T_{N}$ for $\mathrm{BiFeO}_{3}$ with the experimental value of $640 \mathrm{~K}$ shows that for $U \leqslant 5 \mathrm{eV}$, as expected, the mean-field approximation overestimates the magnetic ordering temperature but gives the correct order of magnitude. For $\mathrm{BiCrO}_{3}$ and $\mathrm{Bi}_{2} \mathrm{FeCrO}_{6}$ the ordering temperatures are significantly smaller than for $\mathrm{BiFeO}_{3}$, a fact that can be attributed to the presence of strong $e_{g}-e_{g}$ coupling in the latter compound (see Sec. IV). Therefore, we expect that the magnetic ordering temperature of the recently predicted multiferroic $\mathrm{Bi}_{2} \mathrm{FeCrO}_{6}$ will not exceed $100 \mathrm{~K}$. The calculated $M(T)$ curve for $\mathrm{Bi}_{2} \mathrm{FeCrO}_{6}$ is shown in Fig. 4. We see that no ferrimagnetic compensation temperature (where the two sublattice magnetizations cancel exactly) is observed in the temperature range $0<T<T_{C}$.

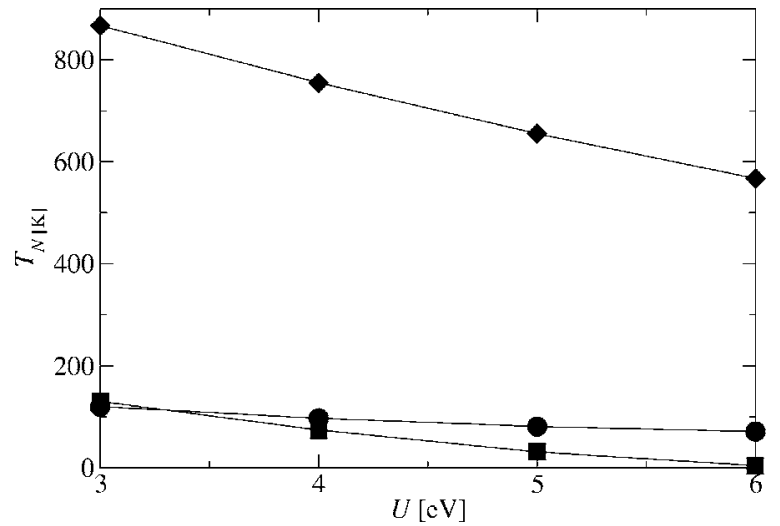

FIG. 3. Magnetic ordering temperatures for structurally relaxed $\mathrm{BiCrO}_{3}, \mathrm{Bi}_{2} \mathrm{FeCrO}_{6}$, and $\mathrm{BiFeO}_{3}$ as a function of the parameter $U$. Circles are for $\mathrm{BiCrO}_{3}$, squares for $\mathrm{Bi}_{2} \mathrm{FeCrO}_{6}$, and diamonds for $\mathrm{BiFeO}_{3}$.

\section{DISCUSSION}

According to Anderson ${ }^{36}$ the superexchange interaction can be separated into two contributions: (i) kinetic exchange which is due to the mixing of the ligand-field orbitals used to describe the spin quasiparticles; this term is proportional to $b^{2} / U$ and is always antiferromagnetic, and (ii) potential exchange which represents the direct exchange interaction between these ligand-field orbitals; this term is always ferromagnetic. Based on this separation, Anderson gave the following simple guidelines for estimating the signs and relative magnitudes of superexchange interactions (note that the same outcomes are reached using the related GoodenoughKanamori rules ${ }^{37}$ ):

(i) Usually, the kinetic exchange is much stronger than the potential exchange and dominates, leading to the predominantly antiferromagnetic interactions found in magnetic oxides.

(ii) The kinetic exchange between $e_{g}$ electrons on different ions connected by a $180^{\circ}$ metal-oxygen-metal bond is much stronger than the kinetic exchange between correponding $t_{2 g}$ electrons, since the former is mediated by $d p \sigma$ bonds whereas the latter is mediated by weaker $d p \pi$ interactions.

(iii) In certain situations the kinetic exchange vanishes by symmetry so that the remaining potential exchange leads to a

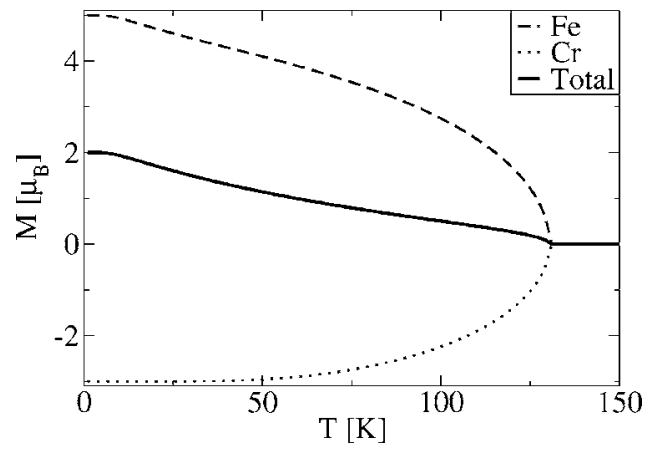

FIG. 4. Calculated temperature dependence of the magnetization for $\mathrm{Bi}_{2} \mathrm{FeCrO}_{6}(U=3 \mathrm{eV})$. The dashed and dotted lines show the magnetizations of the $\mathrm{Fe}$ and $\mathrm{Cr}$ sublattices, respectively. 
TABLE IV. Néel temperatures for different transition metal oxides. All values except for $\mathrm{LaFeO}_{3}$ were taken from Ref. 38).

\begin{tabular}{lc}
\hline \hline Material & $\mathrm{T}_{N}[\mathrm{~K}]$ \\
\hline $\mathrm{MnO}$ & 118 \\
$\mathrm{Fe}_{0.93} \mathrm{O}$ & 198 \\
$\mathrm{CoO}$ & 289 \\
$\mathrm{NiO}$ & 523 \\
$\mathrm{LaCrO}_{3}$ & 282 \\
$\mathrm{LaFeO}_{3}$ & $740^{\mathrm{a}}$ \\
\hline \hline
\end{tabular}

${ }^{\text {aReference } 39 .}$

small ferromagnetic coupling. This occurs for example for a $90^{\circ}$ superexchange coupling between $t_{2 g}$ electrons and also in the case of a $180^{\circ}$ coupling between $e_{g}$ and $t_{2 g}$ electrons.

(iv) Completely filled manifolds, with equal numbers of up- and down-spin electrons give no net contribution to the superexchange interaction.

These rules explain, for example, the relative Néel temperatures of the rock-salt structure transition metal monoxides shown in Table IV. $\mathrm{Ni}^{2+}$ has electron configuration $\left(t_{2 g}^{\uparrow}\right)^{3},\left(t_{2 g}^{\downarrow}\right)^{3},\left(e_{g}^{\uparrow}\right)^{2}$. Therefore, only the two $e_{g}-e_{g}$ interactions contribute to the net superexchange in the $180^{\circ} \mathrm{Ni}-\mathrm{O}-\mathrm{Ni}$ bond (the $t_{2 g}^{\uparrow}-e_{g}$ and $t_{2 g}^{\downarrow}-e_{g}$ interactions are equal and opposite); these are strongly antiferromagnetic, and the Néel temperature is high. For $\mathrm{Mn}^{2+}$, with electron configuration $\left(t_{2 g}^{\uparrow}\right)^{3}$, $\left(e_{g}^{\uparrow}\right)^{2}$, there is an additional ferromagnetic $t_{2 g}-e_{g}$ direct exchange, which weakens the antiferromagnetic coupling and lowers the Néel temperature. $\mathrm{CoO}$ and $\mathrm{FeO}$ are intermediate.

In $\mathrm{BiCrO}_{3}$ the balance of the kinetic and potential exchange leads to a moderate antiferromagnetic coupling between the $t_{2 g}$ electrons on neighboring $\mathrm{Cr}$ sites. This is stronger in the ideal cubic structure than in the distorted $R 3 c$ structure because the perfect $180^{\circ} \mathrm{Cr}-\mathrm{O}-\mathrm{Cr}$ bond angles in the high-symmetry case result in stronger kinetic exchange.

If half of the $\mathrm{Cr}^{3+}$ ions in the cubic structure are replaced by $d^{5} \mathrm{Fe}^{3+}$, the antiferromagnetic part of the nearest neighbor coupling - now between $\mathrm{Fe}^{3+}\left(d^{5}\right)$ and $\mathrm{Cr}^{3+}\left(d^{3}\right)$ - is drastically reduced and, in fact, the interaction becomes ferromagnetic. This can be explained by the additional ferromagnetic interactions between the $e_{g}$ electrons of the $\mathrm{Fe}^{3+}$ and the $t_{2 g}$ electrons of the $\mathrm{Cr}^{3+}$ (see rule iii above). When $U$ is increased, the remaining antiferromagnetic kinetic exchange between the $t_{2 g}$ electrons is suppressed and the net interaction in the cubic structure becomes ferromagnetic. Interestingly, in the relaxed structure of $R 3 \mathrm{Bi}_{2} \mathrm{FeCrO}_{6}$, the nearestneighbor coupling between $\mathrm{Fe}^{3+}$ and $\mathrm{Cr}^{3+}$ is weakly antiferromagnetic. This difference between the cubic and relaxed structures can also be explained within the theory of superexchange: when the structure is relaxed, the $\mathrm{Fe}-\mathrm{O}-\mathrm{Cr}$ bond angle deviates from the perfect $180^{\circ}$, allowing a certain degree of mixing between $\mathrm{Cr} t_{2 g}$ and $\mathrm{Fe} e_{g}$ states. This leads to a nonzero kinetic exchange between these orbitals and a stronger tendency for antiferromagnetic coupling. (Note that similar behavior was discussed in Ref. 40 for the $\mathrm{La}_{2} \mathrm{FeCrO}_{6}$ system.)

Finally, for $\mathrm{BiFeO}_{3}$ the strong $e_{g}-e_{g}$ coupling due to the $d p \sigma$ bonding dominates and results in very strong antiferro- magnetic nearest-neighbor coupling and a high magnetic ordering temperature.

A similar trend in magnetic ordering temperatures than discussed here for the $\mathrm{BiFeO}_{3} / \mathrm{Bi}_{2} \mathrm{FeCrO}_{6} / \mathrm{BiCrO}_{3}$ system can also be observed for the closely related $\mathrm{LaFeO}_{3} / \mathrm{La}_{2} \mathrm{FeCrO}_{6} / \mathrm{LaCrO}_{6}$ system (see Table IV). The ordering temperatures of the end members $\mathrm{LaFeO}_{3}$ and $\mathrm{LaCrO}_{3}$ are comparable to the corresponding $\mathrm{Bi}$ systems but appear to be slightly larger, which could be due to smaller structural distortions in the nonferroelectric La compounds. A Curie temperature of $375 \mathrm{~K}$ and ferromagnetic order were reported for (111)-layered $\mathrm{La}_{2} \mathrm{FeCrO}_{6},{ }^{41}$ but these results are still under debate. ${ }^{40,42}$ From our discussion, ferromagnetic order could be expected for this system, although the reported Curie temperature seems to be too high to be explained by the weak ferromagnetic potential exchange caused by the superexchange interaction.

From the above discussion it can be seen that high magnetic ordering temperatures can be achieved in antiferro-or ferri-magnets by exploiting the strong antiferromagnetic superexchange between $e_{g}$ electrons. The highest ordering temperatures in perovskite structures with octahedral crystal field splittings can occur between two ions of $d^{8}$ electron configuration, although this would of course lead to an antiferromagnet with a net cancellation of magnetic moments. Therefore, we propose that the best choice for a ferroelectric with a net spontaneous magnetization is to combine $d^{5}$ and $d^{8}$ ions. Here, provided that the crystal field splitting is larger than the bandwidth, the material will be insulating, with a net magnetic moment of $3 \mu_{B}$ per formula unit. While other combinations, such as $d^{8}-d^{7}$, would also give strong superexchange coupling, the additional partially filled subshells reduce the probability of insulating behavior. In addition, Jahn-Teller distortions and orbital ordering effects can lead to antiferromagnetic coupling in one direction and ferromagnetic coupling in another direction, which makes the simple rules outlined above more difficult to apply.

Finally, we discuss some possible combinations of elements that will allow $d^{5}-d^{8}$ coupling in the perovskite structure. First we consider retaining $\mathrm{Bi}^{3+}$ as the $A$-site cation in order to exploit its well-understood stereochemically active lone pair to induce the ferroelectric structural distortion. In this case an average oxidation state of $3+$ is required on the cation B site, which limits the possible choices of magnetic ions considerably. $\mathrm{Co}^{4+} / \mathrm{Ni}^{2+}$ would be a possible candidate for strong $d^{5}-d^{8}$ coupling, but it is questionable if such a high oxidation state can be achieved for Co.

Another possibility is to change the oxidation state of the anions, for example, by forming oxyfluorides. For example, $\mathrm{Bi}_{2} \mathrm{Mn}(\mathrm{II}) \mathrm{Ni}(\mathrm{II}) \mathrm{O}_{4} \mathrm{~F}_{2}$ would have the appropriate $d^{5}$ and $d^{8}$ electron configurations and could give the added benefit of an enhanced spontaneous electric polarization by strategic placement of the $\mathrm{F}^{-}$anions. However, the magnetic coupling via $\mathrm{F}^{-}$is weaker than via $\mathrm{O}^{2-}$ and oxyfluorides are generally difficult to prepare and explosive.

A better choice would be to change to a different $A$-site cation, which is still lone-pair active, but which has a higher oxidation state. For example, a $4+A$-site cation would retain charge neutrality with divalent $d^{5} \mathrm{Mn}^{2+}$ and $d^{8} \mathrm{Ni}^{2+}$ on the $B$ sites. In fact, several perovskite systems containing lone-pair 
active $\mathrm{Te}^{4+}$ on the $A$ site have been prepared in the past, ${ }^{43}$ with recent reserch focussing on $\mathrm{Se}_{1-x} \mathrm{Te}_{x} \mathrm{CuO}_{3}$, as a model system to study superexchange interactions. ${ }^{44,45}$ However these systems are usually strongly distorted due to the small size and the stereochemically active lone pair of the $\mathrm{Te}^{4+}$ ion, leading to weak kinetic exchange and small magnetic ordering temperatures. ${ }^{43} \mathrm{Po}^{4+}$ is also a possibility, but since it is radioactive it is less interesting for technological applications.

Moving to the left in the periodic table, $\mathrm{Pb}^{2+}$ and $\mathrm{Tl}^{+}$are also lone pair active cations, and $\mathrm{Pb}^{2+}$ of course is well known as an $A$-site active cation in ferroelectric perovskites. Here, however, the average $B$-site oxidation state must be $4+$, excluding the possibility of a $d^{8}$ configuration. Therefore it is unlikely that room-temperature $\mathrm{Pb}$-based perovskite multiferroics will be identified with this mechanism. Even disregarding its toxicity, the valence of $\mathrm{Tl}^{+}$is even more prohibitive.

In summary, although the spontaneous electric polarization of many perovskite multiferroics persists to temperatures far above room temperature, our analysis suggests that achieving large room-temperature magnetization will continue to be challenging. We suggest $\mathrm{Te}_{2} \mathrm{MnNiO}_{6}$ as one pos- sible candidate within the perovskite structure, but it remains to be seen if the structural distortions in this system are too large to result in strong superexchange coupling. Our analysis indicates that other routes to magnetism, such as the weak ferromagnetism believed to occur in $\mathrm{BiFeO}_{3},{ }^{8}$ are worthy of further pursuit, and that the search for multiferroics should be extended to structures other than perovskite, in which stronger magnetic interactions might be obtained.

\section{ACKNOWLEDGMENTS}

Funding for this work was provided by the National Science Foundation's Chemical Bonding Centers program, Grant No. CHE-0434567, by the American Chemical Society's Petroleum Research Fund, Grant No. 39440-AC5M, and by the MRSEC program of the National Science Foundation, Award No. DMR00-80034. Discussions with Rebecca Janisch and Claude Daul are gratefully acknowledged. N.A.S. acknowledges useful discussions with Dr. Pavel Novak during a visit to the Czech Academy of Sciences supported by the International Center for Materials Research, NSF Grant No. DMR-0409848.
*Electronic address: baettig@mrl.ucsb.edu; Also at Chemistry Department, Université de Fribourg, Pérolles, CH-1700 Fribourg, Switzerland.

${ }^{1}$ N. A. Hill and K. M. Rabe, Phys. Rev. B 59, 8759 (1999).

${ }^{2}$ R. Seshadri and N. A. Hill, Chem. Mater. 13, 2892 (2001).

${ }^{3}$ N. A. Hill, P. Baettig, and C. Daul, J. Phys. Chem. B 106, 3383 (2002).

${ }^{4}$ J. Wang, J. B. Neaton, H. Zheng, V. Nagarajan, S. B. Ogale, B. Liu, D. Viehland, V. Vaithyanathan, D. G. Schlom, U. V. Waghmare, N. A. Spaldin, K. M. Rabe, M. Wuttig, and R. Ramesh, Science 299, 1719 (2003).

${ }^{5}$ S. Niitaka, M. Azuma, M. Takano, E. Nishibori, M. Takata, and M. Sakata, Solid State Ionics 172, 557 (2004).

${ }^{6}$ P. Baettig and N. A. Spaldin, Appl. Phys. Lett. 86, 012505 (2005).

${ }^{7}$ J. B. Neaton, C. Ederer, U. V. Waghmare, N. A. Spaldin, and K. M. Rabe, Phys. Rev. B 71, 014113 (2005).

${ }^{8}$ C. Ederer and N. A. Spaldin, Phys. Rev. B 71, 060401(R) (2005).

${ }^{9}$ F. Bai, J. Wang, M. Wuttig, J. Li, N. Wang, A. P. P. dn A. K. Zvezdin, L. E. Cross, and D. Viehland, Appl. Phys. Lett. 86, 32511 (2005).

${ }^{10}$ P. Baettig, C. F. Schelle, R. LeSar, U. V. Waghmare, and N. A. Spaldin, Chem. Mater. 17, 1376 (2005).

${ }^{11}$ J.-R. Cheng, R. Eitel, and L. E. Cross, J. Am. Ceram. Soc. 86, 2111 (2003).

${ }^{12}$ V. E. Wood and A. E. Austin, in Magnetoelectric Interaction Phenomena in Crystals (Gordon and Breach, New York, 1975).

${ }^{13}$ S. V. Kiselev, R. P. Ozerov, and G. S. Zhdanov, Sov. Phys. Dokl. 7, 742 (1963).

${ }^{14}$ J. R. Teague, R. Gerson, and W. J. James, Solid State Commun. 8, 1073 (1970).

${ }^{15}$ C. Michel, J.-M. Moreau, G. D. Achenbach, R. Gerson, and W. J.
James, Solid State Commun. 7, 701 (1969).

${ }^{16}$ K. Ueda, H. Tabata, and T. Kawai, Science 280, 1064 (1998).

${ }^{17}$ P. E. Blöchl, Phys. Rev. B 50, 17953 (1994).

${ }^{18}$ P. Hohenberg and W. Kohn, Phys. Rev. 136, 864 (1964).

${ }^{19}$ W. Kohn and L. J. Sham, Phys. Rev. 140, 1133 (1965).

${ }^{20}$ G. Kresse and J. Furthmüller, Comput. Mater. Sci. 6, 15 (1996).

${ }^{21}$ G. Kresse and D. Joubert, Phys. Rev. B 59, 1758 (1999).

${ }^{22}$ V. I. Anisimov, F. Aryasetiawan, and A. I. Liechtenstein, J. Phys.: Condens. Matter 9, 767 (1997).

${ }^{23}$ K. Terakura, T. Oguchi, A. R. Williams, and J. Kubler, Phys. Rev. B 30, 4734 (1984).

${ }^{24}$ O. Bengone, M. Alouani, J. Hugel, and P. Blöchl, Comput. Mater. Sci. 24, 192 (2002).

${ }^{25}$ A. Bandyopadhyay, J. Velev, W. H. Butler, S. K. Sarker, and O. Bengone, Phys. Rev. B 69, 174429 (2004).

${ }^{26}$ R. D. King-Smith and D. Vanderbilt, Phys. Rev. B 47, R1651 (1993).

${ }^{27}$ D. Vanderbilt and R. D. King-Smith, Phys. Rev. B 48, 4442 (1993).

${ }^{28}$ F. Kubel and H. Schmid, Acta Crystallogr., Sect. B: Struct. Sci. 46, 698 (1990).

${ }^{29}$ I. Sosnowska, T. P. Neumaier, and E. Steichele, J. Phys. C 15, 4835 (1982).

${ }^{30}$ R. D. Shannon and C. T. Prewitt, Acta Crystallogr., Sect. A: Cryst. Phys., Diffr., Theor. Gen. Crystallogr. 32, 751 (1976).

${ }^{31}$ O. K. Andersen, Solid State Commun. 13, 133 (1973).

${ }^{32}$ V. G. Gavrilyachenko, R. I. Spinko, M. A. Martynen, and R. G. Fesenko, Sov. Phys. Solid State 12, 1203 (1970).

${ }^{33}$ A. H. Morrish, The Physical Principles of Magnetism (WileyIEEE, New York, 2001).

${ }^{34}$ I. V. Solovyev, Phys. Rev. B 65, 144446 (2002).

${ }^{35}$ P. Novák and J. Rusz, Phys. Rev. B 71, 184433 (2005). 
${ }^{36} \mathrm{P}$. W. Anderson, in Magnetism, edited by G. T. Rado and H. Suhl (Academic Press, New York, 1963), Vol. 1, Chap. 2, pp. 25-83.

${ }^{37}$ J. B. Goodenough, Magnetism and the Chemical Bond (Interscience Publishers, New York, 1963).

${ }^{38}$ Landolt-Börnstein-Group III Condensed Matter (SpringerVerlag, Berlin, 2002).

${ }^{39}$ D. Treves, J. Appl. Phys. 36, 1033 (1965).

${ }^{40}$ K. Miura and K. Terakura, Phys. Rev. B 63, 104402 (2001).
${ }^{41}$ T. K. K. Ueda, H. Tabata, Science 280, 1064 (1998).

${ }^{42}$ G. I. Meijer, Science 281, 1571a (1998).

${ }^{43}$ K. Kohn, K. Inoue, O. Horie, and S.-I. Akimoto, J. Solid State Chem. 18, 27 (1976).

${ }^{44}$ M. A. Subramanian, A. P. Ramirez, and W. J. Marshall, Phys. Rev. Lett. 82, 1558 (1999).

${ }^{45}$ J. Iniguez and T. Yildirim, Phys. Rev. B 71, 180415(R) (2005). 